\title{
Edge Dynamics for BF Theories and Gravity
}

\author{
Arshad Momen \\ Department of Physics, Syracuse University \\ Syracuse, NY 13244-1130, U.S.A.
}

\begin{abstract}
We discuss BF theories defined on manifolds with spatial boundaries. Variational arguments show that one needs to augment the usual action with a boundary term for specific types of boundary conditions. We also show how to use this procedure to find the boundary actions for theories of gravity with first order formulations. Possible connection with the membrane approach is also discussed.
\end{abstract}

\section{INTRODUCTION}

Gauge field theories defined on manifolds with boundaries support observables defined on the boundaries called edge states [1]. Their existence can be established from very general arguments and for certain cases they can generate interesting current algebras [2]. These edge variables represent degrees of freedom living on the boundary and can capture a lot of interesting physical information. For example, for condensed matter systems like Quantum Hall samples [3, happen on the boundary, and these edge states provide us with a natural framework to describe the system, when one's attention is focused on the boundary. It is important to 
appreciate in this connection that the nature of the edge degrees of freedom is not completely arbitrary but is rather constrainted by the bulk dynamics and therefore can reflect the properties of the bulk.

Recently, we managed to establish the existence of such states also in gravity, when one excises a certain part of spacetime, for instance, a black hole [6]. These states then have support on the boundary of the spacetime region that has been cut out. Like their condensed matter analog, they can give important clues to our understanding of black hole physics, for instance, the origin of black hole entropy [7]. In this connection, in a previous paper we managed to show in a toy model in $(2+1)$ dimensions that the entanglement entropy [8] one gets after tracing out the edge degrees of freedom scales with the perimeter ( the "area law") in the weak coupling limit [9]. This result definitely encourages one to look for a similar situation in four dimensions which might be of relevance for black hole physics.

Given the existence of these variables, an important puzzle is the dynamics of these edge variables in term of an action on the boundary. The solution to this question is partially known for certain systems, e.g. three dimensional gauge theories which include the ChernSimons term [10, 1]. In this paper, we will show that similar results can be obtained for field theories which contain the so-called topological BF term. This will shed light on the problem of constructing the action for gravitational edge states.

The pure BF theory [11, 12] is known to be a topological field theory. Unlike the ChernSimon action which can be defined only in three dimensions, one can define the BF action in any spacetime dimension. This action has appeared in various forms in different contexts, being responsible for novel statistics for stringy objects [13] or the theory of Josephson junction arrays [14] as well as for the classification of 2-knots [15]. The form of the BF action is very much similar to the first order formulations of gravity like the Palatini formalism [16] or the self-dual ( or Ashtekar ) formalism [17]. In fact, one particular motivation for this investigation was to find the dynamics for the edge states appearing in gravity [6]. The results obtained here can be applied to gravity theories, specially to two-form gravity theories [18]. 
The paper is arranged as follows. In section 2, we review how the presence of the boundary leads us to a boundary action for the edge variables where the bulk dynamics is governed by a Chern-Simons theory. In section 3, we will follow similar steps to construct a boundary action for the abelian BF theory defined on $3+1$ dimensional manifolds. In section 4, we extend our results to the non-abelian case. Next, in section 5, we show how to write a boundary action for gravity in one of its first order formulations. Finally, We discuss the possible uses of the boundary action.

We will be using the differential form notation extensively in this paper though at times we will use the component notation also.

\section{CHERN-SIMONS ACTION WITH BOUNDARY}

For orientational purposes, in this section we will describe the abelian case only. However, the extension to the non-abelian case is fairly straightforward. The treatment given below is also quite standard in effective theories of the Quantum Hall Effect [19,20, 1 ,

Let our spatial slices be diffeomorphic to a disk $D$, so that the spacetime $\mathcal{M}$ is $D \times \mathbb{R}$. The Chern-Simons action defined on this three-dimensional manifold which has a boundary $\partial \mathcal{M}=S^{1} \times \mathbb{R}$ is given by

$$
S_{0}=\frac{k}{4 \pi} \int_{\mathcal{M}} A \wedge d A
$$

Because of the boundary, this action is not invariant under a gauge transformation

$$
A \rightarrow A+d \Lambda
$$

as it picks up the surface term

$$
S_{0} \rightarrow S_{0}-\frac{k}{4 \pi} \int_{\partial \mathcal{M}} d \Lambda \wedge A
$$

$\partial \mathcal{M}$ being $\partial D \times \mathbb{R}=S^{1} \times \mathbb{R}$. If we require that the system be described by a gauge invariant

action, then we must add a surface term to the Chern-Simons action $S_{0}$ to get an action $S$ as follows: 


$$
\begin{aligned}
S_{t o t} & =S_{0}+\frac{k}{4 \pi} \int_{\partial \mathcal{M}} d \phi \wedge A-\alpha \int_{\partial \mathcal{M}} D \phi \wedge * D \phi, \\
D \phi & \equiv d \phi-A .
\end{aligned}
$$

Under the gauge transformation, (2.2), $\phi$ simultaneously transforms as

$$
\phi \rightarrow \phi+\Lambda
$$

rendering (2.4) gauge invariant.

One can think of $\phi$ as the phase of a complex Higgs field, $\Phi=\rho e^{i \phi}$, where the amplitude $\rho$ is frozen to $\rho=v$.

Note that we have added a kinetic energy term for the field $\phi$. The parameter $\alpha$ is a constant. This term is not needed for gauge invariance of (2.4), but gives interesting dynamics to $\phi$.

So far, the coefficient $\alpha$ in (2.4) has remained arbitrary. However, if this action were to describe QHE [4], then the currents due to the edge scalar field $\phi$ are required to be "chiral" [20]. For instance, if we demand that the currents are left-moving, the condition one should impose is

$$
D \phi-* D \phi=0
$$

This fixes the coefficient $\alpha$ to be

$$
\alpha=\frac{k}{8 \pi}
$$

Thus the correct action describing QHE is

$$
S_{\text {tot }}=S_{\text {bulk }}+\frac{k}{4 \pi} \int_{\partial \mathcal{M}} d \phi \wedge A-\frac{k}{8 \pi} \int_{\partial \mathcal{M}} D \phi \wedge * D \phi
$$

We will show below that analogous actions can be constructed for the BF systems as well.

It is interesting to note that boundary actions, similar to the above have been constructed for the non-abelian Chern-Simons case by variational arguments [21] and gauge arguments [6] also. 


\section{ABELIAN BF THEORY}

We will now discuss how to write boundary actions for BF type field theories defined on manifolds with spatial boundaries. The constraint analysis for such field theories is discussed in [5] where it is shown that they too contain edge states [5]. In contrast to these papers, which focus on the canonical approach, we will stress variational arguments here. They can be applied to the case of gravity too.

Let us begin with a $3+1$ dimensional spacetime $\mathcal{M} \equiv \mathbb{R} \times \Sigma$ where the spatial part $\Sigma$ is a manifold with the boundary $\partial \Sigma$. So, the boundary of the spacetime is $\partial \mathcal{M} \equiv \mathbb{R} \times \partial \Sigma$. The action describing the BF system is

$$
S_{0}=\int_{\mathcal{M}} B \wedge F=\frac{1}{2} \int_{\mathbb{R} \times \Sigma} d t d^{3} x \epsilon^{i j k l} B_{i j} \partial_{k} A_{l}
$$

where $B=\frac{1}{2} B_{i j} d x^{i} d x^{j}$ is a two-form and $F=d A=\partial_{j} A_{k} d x^{j} d x^{k}$ is the curvature form constructed out of the connection form $A$.

The equation of motion for $A$ can be obtained by varying the $B$ field in the bulk and is

$$
F=0 \text {. }
$$

Notice that the equation of motion and the action as well are trivially invariant under the gauge transformation

$$
A \rightarrow A+d \Psi
$$

where $\Psi$ is a zero-form.

To get the equation of motion for $B$ one has to vary $A$. Under a generic variation of $A$, the variation of the action is given by

$$
\delta S_{0}=\int_{\mathcal{M}}[B \wedge d(\delta A)]=\int_{\mathcal{M}}[-d B \wedge \delta A]+\int_{\partial \mathcal{M}}[B \wedge \delta A]
$$

The sign for the boundary term is fixed by the orientation in the bulk : $\epsilon^{t i j k}=\epsilon^{i j k}$ where $\epsilon^{123}=1$.

So, there is a surface term in $\delta S$ for a generic variation of $A$. We wish to have such a surface term equal to zero. This can be achieved in any one of the following ways: 
1. The variation $\delta A$ is chosen to vanish at the boundary,

$$
\left.\delta A\right|_{\partial \mathcal{M}}=0
$$

2. One can choose $B$ to be zero on the boundary,

$$
\left.B\right|_{\partial \mathcal{M}}=0
$$

3. One also has the option of adding surface terms.

The first two options are no good when nontrivial edge dynamics are sought for. As for the last option, one can augment the action $S_{0}$ with the surface term

$$
S^{\prime}=-\int_{\partial \mathcal{M}} B \wedge A
$$

The option we would be exploring now for the augmented action $S_{0}+S^{\prime}$ is to vary $B$ in the bulk without changing its boundary value which can be nonzero :

$$
\begin{array}{r}
\left.\delta B\right|_{\partial \mathcal{M}}=0, \\
\left.B\right|_{\partial \mathcal{M}}=\text { Not necessarily zero. }
\end{array}
$$

This is different from (3.6). The choice of this type of boundary condition can be motivated from the fact that $A$ is a connection whereas $B$ is not.

When one looks at the augmented action

$$
\begin{aligned}
S & =S_{0}+S^{\prime}=\int_{\mathcal{M}} B \wedge F-\int_{\partial \mathcal{M}} B \wedge A \\
& =\int_{\mathcal{M}} A \wedge d B,
\end{aligned}
$$

one sees that this is nothing but the London action

$$
S=-\int_{\mathcal{M}} * j \wedge A
$$

with the dual identification $j=* d B$ of the current [5]. But, when one has other terms in the action which involve the coupling of the $B$ field to other fields, evidently such a simple interpretation is not necessarily available. 
The equation of motion for $B$ following from (3.9) is

$$
d B=0
$$

The action (3.9) now enjoys the gauge invariance

$$
B \rightarrow B+d \chi
$$

where $\chi$ is a one-form.

It is interesting that the action $(3.9)$ can also be constructed by demanding invariance of the action under the transformation (3.12) alone without using the boundary conditions on $B$.

The modified action (3.9) has lost its invariance under the usual gauge transformation

$$
A \rightarrow A+d \Lambda
$$

To remedy this situation, we introduce a scalar field $\Phi$ on the edge ( i.e. on the boundary ) transforming as

$$
\Phi \rightarrow \Phi-\Lambda
$$

so that the combined one-form

$$
A^{\prime} \equiv A+d \Phi=D \Phi
$$

remains invariant under the gauge transformation. Given these quantities, we can write down the following gauge invariant action inspired by our previous experience with the Chern-Simon case :

$$
\begin{aligned}
S_{\text {tot }} & =\int_{\mathcal{M}}[B \wedge F]-\int_{\partial \mathcal{M}}\left[B \wedge A^{\prime}+\lambda\left(A^{\prime} \wedge * A^{\prime}\right)\right] \\
& \equiv \int_{\mathcal{M}} B \wedge F+S_{\text {boundary }} .
\end{aligned}
$$

Here the Hodge $*$ operation is defined with respect to the boundary metric and $\lambda$ is a constant with mass dimension 1 . 
We would like to stress that as this action was found by fixing the boundary value of $B$, we are not allowed to make a variation of $B$ on the boundary to get the equations of motion on the boundary. However, one is allowed to vary $A$ or $\Phi$ at the boundary. Varying $\Phi$ one gets the equation of motion for $\Phi$ on the boundary:

$$
d(\lambda * D \Phi-B)=0
$$

while varying $A$ one gets

$$
\frac{\delta S}{\delta A}=0 \Rightarrow \lambda D \Phi=0
$$

Compatibility with the equation (3.18) then lead to the condition

$$
d B=0 \Rightarrow B=d \xi
$$

that is on the boundary $B$ is to be a "pure gauge".

The total action (3.16) can also be motivated from the boundary theory. This can be seen as follows. A charged scalar field $\Phi$ coupled to the antisymmetric field $B$ is described by

$$
S_{\text {boundary }}=\int_{\partial M}\left[\lambda(d \Phi+A)^{*}(d \Phi+A)+(d \Phi+A) \wedge B\right]
$$

However, varying $B$ in above equation we get the condition

$$
D \Phi=0
$$

on the boundary. On the other hand varying $A$ we are led to

$$
\frac{1}{2} * \lambda(D \Phi)+B=0
$$

It must therefore be that there exists another term such that the term involving the variation of $A$ on the boundary involving the $B$ field exactly cancels out. This, as can easily be seen, turns out to be the BF term defined in the bulk. Hence, the action (3.16) hangs together neatly. This picture is basically the same as the one where one can argue for the existence of 
the Chern-Simons action on a disk starting from the edge action for a gauged chiral scalar field theory [19:团.

One can easily show that the above picture is applicable to arbitrary spacetime dimensions.

\section{NON-ABELIAN BF SYSTEM}

Given the above construction for edge dynamics for the abelian BF system, one can generalize it to non-abelian BF systems also. For the non-abelian case, the two-form $B$ and the connection $A$ are valued in a Lie algebra $G$. We also assume that this Lie algebra is endowed with an invariant trace, which we denote by $\mathrm{Tr}$. The BF action is then described by

$$
S_{0}=\int_{\mathcal{M}} \operatorname{Tr} B \wedge F
$$

where the curvature form $F$ is now given by

$$
F=d A+A \wedge A
$$

The equation of motion arising from the variation of $B$ in the bulk is

$$
F=0
$$

However, a surface term like the one in (3.4) appears when one tries to vary $A$. Therefore, as before we have to add a boundary action

$$
S_{b}=-\int_{\partial \mathcal{M}} \operatorname{Tr} B \wedge A
$$

to allow for a non-zero boundary value for $B$.

We assume that the gauge transformation for $A$ now takes the standard form of the non-abelian connection :

$$
A \rightarrow g A g^{-1}+g d g^{-1},
$$


while $B$ transforms as

$$
B \rightarrow g B g^{1} .
$$

Note that $S_{0}+S_{b}$ is not invariant under (4.5)). So, we introduce an edge field transforming as

$$
h \rightarrow g h
$$

in order that one can construct the one-form

$$
h^{-1} D h=h^{-1}(d+A) h \equiv A^{\prime}
$$

invariant under the $g$ transformation. Proceeding as before, the complete action can be determined as

$$
S_{\text {tot }}=\int_{\mathcal{M}} \operatorname{Tr}[B \wedge F]-\int_{\partial \mathcal{M}} \operatorname{Tr}\left[B \wedge A^{\prime}+\lambda\left(A^{\prime} \wedge^{*} A^{\prime}\right)\right]
$$

The constraint that follows by varying $A$ is given by

$$
\lambda h^{-1} D h=0 .
$$

while the equation of motion for the $h$ field turns out to be

$$
d\left(* \lambda\left(h^{-1} D h\right)-B\right)=0 .
$$

Compatibility of (4.10,4.11) requires

$$
d B=0
$$

on the boundary. as in the abelian case. However, this boundary equation is not invariant under the transformation (4.6).

\section{AN ALTERNATIVE ACTION IN FOUR DIMENSIONS}

The construction shown in previous sections suffers from an aesthetically unpleasant feature, namely the introduction of the Hodge $*$ operation which requires the definition 
of a metric on the boundary. This prevents the field theory on the boundary from being a"topological" theory. However, when we are working in four dimensional bulk spacetime so that the boundary happens to be a three dimensional surface, one can write a ChernSimons action on the boundary which does not require any metric. So, the complete theory is diffeomorphism invariant and a topological field theory as well!

So, the complete action in the latter case would be given by

$$
S_{\text {total }}=\int_{\mathcal{M}} B \wedge F-\int_{\partial \mathcal{M}}\left[B \wedge A^{\prime}+\left(A^{\prime} d A^{\prime}+\frac{2}{3} A^{\prime} \wedge A^{\prime} \wedge A^{\prime}\right)\right]
$$

where $A^{\prime}=h^{-1} D h$ as in eq. (4.8).

As before we use also the boundary action, consisting of the terms defined on the boundary in the above equation, to get the equation by varying $A$, namely,

$$
\left.F\left(A^{\prime}\right)\right|_{\partial \mathcal{M}}=0
$$

\section{THE TREATMENT FOR GRAVITY}

It is well-known that the vacuum Einstein equations can be obtained from the variation of the first order Palatini action ( also known as Einstein-Cartan action for our case) given by

$$
S_{\text {grav }}=\int_{\mathcal{M}} d^{4} x \epsilon_{a b c d} \epsilon^{\mu \nu \rho \lambda} e_{\mu}^{a} e_{\nu}^{b} R_{\rho \lambda}^{c d} \equiv \int_{\mathcal{M}} \operatorname{Tr}(e \wedge e \wedge R)
$$

where $e_{\mu}^{a}$ are the components of the tetrad and $a, b, .$. are the indices indicating that the object is valued in the $S O(3,1)$ Lie algebra. The curvature tensor is obtained from the spin connection:

$$
R_{a b}=d \omega_{a b}+\omega_{a}^{c} \wedge \omega_{c b}
$$

To obtain the equation of motion, one varies the tetrad and the spin connection independently. Note that this action is very similar to the non-abelian BF action, the important difference being that one has the two-form $B$ replaced by the two-form $e \wedge e$ constructed 
out of the one-form $e$. So, one does not have the extra gauge invariance (3.14) for the $B$ field anymore. However, this does not deter us from proceeding in a fashion similar to the BF case using the variational arguments. In fact there exist formulations of gravity [18,22 where one replaces the Einstein-Cartan action by a BF like action augemented by an extra term which basically enforces the algebraic condition $B=e \wedge e$. These extra terms do not involve the spin connection. One sees that the analog of (3.8) now is

$$
\left.\delta e\right|_{\partial \mathcal{M}}=0
$$

which tells us that we are fixing the metric on the boundary. This boundary can be the (stretched) horizon of the black hole.

Varying $\omega$, we find the boundary term

$$
\int_{\partial \mathcal{M}} E \wedge E \wedge \delta \Omega
$$

where $E$ and $\Omega$ are the pullbacks of the tetrad $e$ and the spin connection $\omega$ respectively to the boundary $\partial M$. Therefore, the term to be added to the action is

$$
\int_{\partial \mathcal{M}} \epsilon_{a b c d} E^{a} \wedge E^{b} \wedge \Omega^{c d}
$$

Like for gauge invariance in the BF case, the boundary term is not invariant under the local Lorentz transformation

$$
\Omega \rightarrow \Lambda \Omega \Lambda^{T}+\Lambda d \Lambda^{T}
$$

where $\Lambda$ is valued in the $\mathrm{SO}(3,1)$ group (in its $4 \times 4$ irreducible representation), so $\Lambda_{\mu}^{\alpha} \Lambda_{\nu}^{\beta} \eta_{\alpha \beta}=$ $\eta_{\mu \nu}$. This situation can be remedied by replacing the connection form $\Omega$ with the one-form

$$
\Omega^{\prime} \equiv u^{T}(d+\Omega) u
$$

which is invariant under a local Lorentz transformation provided $u$ is in the same representation as $\Lambda$ and transforms as

$$
u \rightarrow \Lambda u
$$


under this transformation. Note that the above construction is absolutely parallel to the construction (4.8) in the non-abelian BF theory. One can see that the modified action is

$$
S_{\text {mod. }}=\int_{\mathcal{M}} \operatorname{Tr}(e \wedge e \wedge R)+\int_{\partial \mathcal{M}} E \wedge E \wedge \Omega^{\prime}
$$

The boundary term in above action has appeared in a different context. This type of action describes the coupling of extended objects to external gauge fields [23]. This boundary action would then describe the coupling of a membrane to a non-abelian connection. It may be that a stertched membrane description [24,25] arises naturally from this "gauge degrees"

on the boundary. In fact, a similar picture emerges in the case of the $2+1$ dimensional black hole [7].

In this treatment, we have fixed $e$ and hence the metric on the boundary, and this could be a black hole metric too. It is interesting to note that then this shows that there are gauge degrees of freedom living on the boundary of the black hole in the same sense as in [6, (]].

One can also add a Chern-Simons action constructed from the one-form $\Omega^{\prime}$, as in section $\mathrm{V}$, to the action (6.9). The constraint one gets by varying $\Omega^{\prime}$ is

$$
\left.R\left(\Omega^{\prime}\right)\right|_{\partial M}=0
$$

This boundary condition is interesting. This tells us that one is really interested in the flat $S O(3,1)$ connections on the boundary. However, the boundary of spacetime has the topology $\mathbb{R} \times \Sigma$ where $\Sigma$ is a surface surrounding the black hole.

The moduli space of the flat connections valued in the Lie algebra of compact groups are known to be finite dimensional. Though $S O(3,1)$ is not compact, a compact subgroup of it is all that we might be interested in and this might of relevance to the finiteness of the black hole entropy.

\section{CONCLUSIONS}

In this note, we have derived an action for the edge variables whose bulk dynamics is given by a four-dimensional BF theory. Note that in our approach it is not necessary that the 
field theory in the bulk is given by a pure BF theory. One can add terms like the integral of $B \wedge B$ to the bulk action. So, the above analysis can be applied very easily to the first order formalisms for gravity. In fact, one notices that in gravity the two-form $B$ can be thought of as $e \wedge e$ where $e$ is the tetrad one-form. Hence, naively speaking, the boundary condition for $B$ fixes the metric on the boundary ( which can be null as well). The boundary term needed to make the variation well-defined is not invariant under the local $\mathrm{SO}(3,1)$ transformations showing that there are gauge degrees of freedom on the boundary.

It would be instructive to see whether the above states give rise to an entropy with interesting properties like their three dimensional counterpart [7].

\section{ACKNOWLEDGMENTS}

I would like to thank my advisor Prof. A.P. Balachandran for his valuable guidance, support and constant encouragement. I would also like to thank J. Goldberg, T.R. Govindarajan, D. Marolf, V. John and S. Vaidya for discussions. I am specially grateful to L. Chandar who participated in the initial stages of this work. I am indepted to Steve Carlip, S. Major and Siddharta Sen for providing valuable criticisms on earlier versions of this paper. Part of this work was completed during TASI'96 and I would like to the organizers for their hospitality. This work was supported in part by the US Department of Energy under the contract number DE-FG02-85ER40231. 


\section{REFERENCES}

[1] For a overview see, A.P. Balachandran, L. Chandar and E. Ercolessi, Int. J. Mod. Phys. A 10(1995)1969.

[2] E. Witten, Comm. Math. Phys. 121 (1989)351.

[3] X.G.Wen, Topological orders and edge excitation in FQH states(cond-mat/9506066).

[4] A. P. Balachandran, L. Chandar, B.Sathiapalan, Nucl. Phys. B 443(1995)465.

[5] A.P. Balachandran, P. Teotonio, Int. J. Mod. Phys. A 8 (1994)723, ibid.A 9 (1994)1569.

[6] A.P. Balachandran, L. Chandar and A. Momen, Nucl. Phys. B 461(1996)587.

[7] S. Carlip, Phys. Rev. D 51(1995)632.

[8] L. Bombelli, R. Kaul, J. Lee and R. Sorkin, Phys. Rev. D 34 (1986)373, M. Srednicki.

[9] A.P. Balachandran, L. Chandar, A. Momen, preprint- SU-4240-617 (hep-th/9512047).

[10] See for example, J. Fröhlich and U.M. Studer, Rev. Mod. Phys. 65 (1995)733 and references therein.

[11] G. Horowitz, Comm. Math. Phys., 125(1989)417.

[12] M. Blau and G. Thompson, Ann. Phys. 205(1991)130.

[13] C. Aneziris, A.P. Balachandran, L. Kauffman and A.M. Srivastava, Int. J. Mod. Phys. A 6(1991)2519.

[14] M. Diamantini, P. Sodano and C. Trugenberger, Nucl. Phys. B 448(1995)505, CERN preprint CERN-TH-95-294(hep-th/9511168).

[15] A. Cattaneo, P. Cotta-Ramusino, J. Fröhlich and M. Martellini, Jour. Math. Phys. 36(1995)6137 and reference s therein.

[16] See P. Peldan, Class. and Quant. Grav. 11(1994) 1087 for a review of various actions 
in gravity.

[17] J. Samuel, Pramana 28(1987)L429 and L. Smolin and T. Jacobson, Phys. Lett. B 196 (1987)39. For an extensive discussion see A. Ashtekar, New Perspectives on Canonical Gravity, Bibliopolis, Napoli, 1991.

[18] R. Capovilla, J. Dell, T. Jacobson and L. Mason, Class. and Quant. Grav. 8 (1991) 41; R. Capovilla, J. Dell and T. Jocobson, ibid. A 8 (1991) 59.

[19] C.G. Callan and J. Harvey, Nucl. Phys. B 250 (1985) 427, S. G. Naculich, Nucl. Phys. B 295 (1988) 837.

[20] See for example F. Wilczek in Fractional Statistics and Anyon Superconductivity, ed. F. Wilczek, World Scientific, Singapore, 1990; M. Stone, Ann. Phys. 207(1991)38. The microscopic arguments originates in B. Halperin, Phys. Rev. B 25 (1984) 2165.

[21] S. Carlip, Nucl. Phys. B 362 (1991)111. See also in a related context, P. Salomonson, B. S. Skagerstam and A. Stern, Nucl. Phys. B 342(1990) 769.

[22] G. 't Hooft, Nucl. Phys. B 357(1991)211, I. Bengtson, Class. Quantum Grav. $12(1995) 1581$.

[23] A. P. Balachandran, A. Stern and B. S. Skagerstam, Phys. Rev. 20 (1979) 439; A. P. Balachandran, G. Marmo, B. S. Skagerstam and A. Stern, J. Phys. G 7(1981)1001; A. P. Balachandran, V. P. Nair B. S. Skagerstam and C. G. Trahern, Phys. Rev. D 26 (1982)1442.

[24] K. S. Thorne, R.H. Price and D. A. Macdonald, Black Holes : The Membrane Paradigm, Yale University Press (1986).

[25] M. Maggiore, Phys. Rev. D 49 (1993) 2918; Phys. Lett. B 333(1994)93, ibid. 341(1995)450(E); Nucl. Phys. B 429 (1994)205 and references therein. 